\title{
Concluding Remarks: Playing by the Numbers
}

\author{
Virginia Trimble \\ Department of Physics and Astronomy, University of California Irvine, \\ Irvine, CA 92697-4575, USA \\ And \\ Las Cumbres Observatory \\ Goleta, CA 93117, USA \\ vtrimble@uci.edu
}

\begin{abstract}
Advances in available computing facilities, judiciously employed by our colleagues, have undoubtedly enhanced our understanding of the processes by which stars (and planets) form, from very diffuse gas to something you could almost live around. Nevertheless we remain very far from being able to describe (let alone explain) what is going on in many cases. And this is likely to remain true even as Moore's Law growth begins to hit its head against energy and power considerations. A large fraction of long-standing questions appear to have honest answers of the general form, "yes and no", "all of the above", or "some of them are and some of them aren't". This includes many of my favorites, like triggering, formation of binary populations, and the role of magnetic fields. Rather few questions have actually been retired from the universe of discourse in recent years.
\end{abstract}

\section{Introduction}

I arrived at the meeting with two lists of questions: a fairly long one of items I had been wondering about before looking at the abstracts, and a considerably longer one (generally addressing details) derived from the titles and abstracts, especially of the posters. Most of these questions have been around for a decade or more. Michael Norman suggested that progress can be gauged by the number and importance of the questions that can be retired from discussion, in the sense, for instance, that we no longer ask (with Simon Newcomb a century ago) what is the primary source of stellar energy. Richard Larson took us briefly back to the era, preceding and surrounding World War II, when it was by no means certain that ongoing star formation was either real or important. This, he pointed out, has definitely been settled, in favor of the process and its early supporters, including Lyman Spitzer.

A good many other classic questions can be retired, but only in the sense that the answer is, for instance, that there is triggering by more than one mechanism, that massive stars grow in several different ways, and that both turbulence and magnetic fields are important, often at the same time. The questions then return in the form of which happens when and where, and why, invariably demanding a good deal more computing power to try to follow everything at once.

The following sections include a few historical remarks, my lists of questions in, at best, semi-ordered form, followed by summaries of a few specific subfields, and a conclusion of a sort. 


\section{A Bit of History}

Early computational tools included the pencil and the abacus (though the ones shown at the symposium were not used either by Jeans - it was an American pencil - or by Hayashi in establishing his tracks - it was a Chinese abacus, and in any case he had access to electronic computing facilities, including those at Goddard Space Flight Center. (Sugimoto 2010). Computers were, of course, once people, generally women with some college education who processed data for the male observers. The best known at Harvard included Henrietta Swan Leavitt (Cepheids), William Fleming, Antonia Maury, and Annie J. Cannon, whose spectral types were, respectively, too simple, too complex (including a criterion that separated out giants to which Pickering did not subscribe), and just right. Cecilia Payne Gaposchkin was their contemporary, but a modern, full-fledged researched astronomer, who asked difficult questions and managed to answer some of them.

Sir James Jeans was a pioneer of the pencil and paper school, who had a mass and a length, and served as president of the Royal Astronomical Society for two year ending in 1927 (keep that date!). He only rather gradually accepted the existence of other galaxies and the "short" time scale $\left(10^{10} \mathrm{yr}\right)$ for the universe, as against his own $10^{12}-10^{13} \mathrm{yr}$ required for galaxies, star clusters, and binary systems to achieve their current appearance using processes he had thought of. Thus for much of his career, he opined that (a) gaseous spirals became star clusters, (b) rotating gaseous spheres became Maclaurin spheroids, then Jacobi ellipsoids (with the RV Tauri stars as possible examples)and then close binaries, while (c) encounters were needed to account for wide binaries and planetary systems, since the "nebular" hypothesis (to which we all now subscribe) would leave $90 \%$ of the angular momentum in the sun. He wrote, first, (Jeans 1933) that by the time "the whole of the gas is condensed into detached globules, calculation shews that each globule would have about the same mass as an actual star, and, second, that if you start with the entire universe as a chaotic mass of gas, finally the whole mass would condense or break up, into detached masses of denser gas (for which) calculations shew that these would be on something like the scale of the actual nebulae, and would form at about the average distance apart of the observed nebulae, all of about the same size, weight, and intrinsic brightness. This is found very approximately to be the case." The observations he had in mind must have been overwhelmingly dominated by selection effects!

Later computational tools included punch cards for both Fortran commands and data, output on oversized, green-lined paper, and the Christy code, mentioned by Richard Larson, which I applied to look for Cepheid-type pulsations of R CorBor stars (yes they do) in 1970-71. Near the beginning of this stage Hayashi (1961) showed that stars condensing out of diffuse gas would be completely convective and so follow tracks in the HR diagram sharply upward and then down onto the main sequence (Hayashi tracks!) rather than sneaking in from the lower right hand corner under radiative equilibrium as had been previously thought.

Abandonment of the encounter hypotheses for planetary system formation and establishment of the Hayashi tracks count as progress in star formation (in Norman's sense) over, roughly, the last 70 years. Table 1 describes progress over about the last 40 years, in the sense that some things just barely calculable then have become routine now, while the topic of galaxy formation has advanced from incomprehensible to, at least, respectable. That item violates my general principle of mentioning very few speakers and almost no poster presenters by name, because those names, in order in their sessions, so conveniently give us the A, B, C of the subject. TLA, in case you weren't sure, is a Three Letter Acronym. In addition, formation of galaxies and stars (at least the first generation) 
Table 1. Progress in Computability of Formation and Evolution of Stars and Galaxies.

\begin{tabular}{|l|c|c|}
\hline Topic & New Code, 1968 & Now \\
\hline Galaxy Evolution & Tinsley (1968), top down & Bottom up \\
\hline Star Evolution & Paczyński (1970) & On a disk near you \\
\hline Star Formation & Larson (1969) & AMR, SPH, TLA, etc. \\
\hline Galaxy Formation & - & | Abel, Bournaud, Colin, and all \\
\hline
\end{tabular}

are no longer so separable as we once thought them. The topic of galaxy evolution has gone through a paradigm shift from "top down" to "bottom up." Tinsley's (1968) initial conditions had a gas cloud with mass equal to the eventual galaxy, which was turned into stars in, perhaps, 10 time steps, with fixed IMF, and the nuclear products then dumped back into the remaining gas. It had a "G dwarf problem" but otherwise yielded quite a decent fit to the range of galaxy luminosities, colors, compositions, and residual gas fractions that we see. Galaxy evolution in 2010 proceeds largely through star formation in dwarf entities intermingled with mergers and captures, which tend to trigger more star formation, with the results being required to look not so very different from Tinsley's.

Also more or less historical, I suppose, is a decoding of the hieroglyphic writing of the name of the NUT project, which combines more aspects of Egyptian orthography than one usually sees in one place. The three water jugs and the sky at the beginning associate her with rain. The three jagged lines are N's, giving the plural N-W (which might alternatively have been written with $\mathrm{N}$ plus the "weak bird" glyph, whose sound is W). The loaf of bread is a phonetic T, so that we now have her name "spelled out". Then comes the distorted oval shaped womb, indicating her association with childbirth, and finally the figure of a goddess, so that the final ordering is determinatives-alphabetic writing-more determinatives.

\section{My Initial Question}

Some of these can indeed be phrased as questions (Is star formation triggered? for instance). But most seem to look more natural as parts of a list of things we would like to understand, and are shown that way here.

Physics to be included and initial conditions: Gravity is the sine qua non of structure formation, though my initial list left it out (by accident), and at least one speaker claimed (deliberately) that it wasn't needed. Other items are distributions of temperature and density, ionization (due primarily to cosmic rays inside molecular clouds), the strength and structure of magnetic fields and of turbulence, and the amount and composition of dust.

Molecular clouds, sometimes giant ones (GMCs) are the locus of star formation, at least here and now. Thus "why do stars form?" begins with what assembles GMCs; what causes them to start collapsing or fragmenting; how efficient is the process, and what does this efficiency depend on; and how do stars form in environments very different from a temperature around $10 \mathrm{~K}$ and a density of $10^{3-5} \mathrm{Hcm}^{-3}$.

Triggering is generally thought to be part of why collapse and fragmentation begin, but if it happens only some of the time, what difference does it make (to efficiency, the IMF, or whatever). On global scales, mergers, infalling dwarfs, and other encounters 
are possible triggers. More locally, we might have shock waves at spiral arms (hardly mentioned at the meeting), cloud-cloud collisions, expanding HII regions and supernova remnants, UV radiation, and other shock sources.

Clusters of stars are the natural outcome of fragmenting GMCs, but we are not quite certain that all stars form in clusters, even very short-lived ones. One would like to know the range of final cluster masses (in stars) vs. the initial gas cloud mass, and also, within any one cluster, how co-eval are the stars, is there an ordering of formation by star mass or propagation of star formation in space, and is mass segregation primordial or dynamical, this last a case that demands the answer, both please!

The equivalent of dynamic range remains a problem. A decade ago, it was possible for a theorist to start with a cloud of $50 M_{\odot}$ and follow it fairly honestly down to the scale of binaries and protoplanetary disks (Alves \& McCaughrean 2001). Naturally very few $100 M_{\odot}$ stars resulted. I arrived wondering how much the treatable mass had increased. Two different presentations said 500 and $1000 M_{\odot}$ (just right for the number of Moore's law doubling times, though not yet quite an $\mathrm{OB}$ association).

Stellar populations formed under very different conditions might well be expected to differ from the disk of the Milky Way. One would particularly like to know just what Population III was like and where their remnants might be lurking (IMBHs in the cores of globular clusters??). Other somewhat mysterious contexts include youngish stars in mostly old galaxies (formed in situ or brought in by mergers?) and the condition under which globular clusters form, both past and present. They aren't making them any more, at least in our galaxy.

The initial mass function looks more similar from place to place than one might have guessed. One would like observations of it in regions of different metallicity, cloud turbulence, magnetic fields, and redshift, and calculations that can match the full range found. A topic of some dispute in recent years has been the extent to which the IMF is already "coded in" at the stage of starless cores in molecular clouds.

Stars of more than about $10 M_{\odot}$ would seem to have difficulty in assembling by accretion (because their expected luminosities rise above the Eddington limit). Thus one asks whether they can fool Mother Nature, whether some other process is required (mergers of less massive cores, presumably), or both. Similar questions arise for the formation of brown dwarfs and orphan planets (not because of Eddington limit, but because there doesn't seem to be anything obvious to stop them accreting). Again, several processes have been suggested, and if they all contribute, one is surprised at the relative uniformity of the IMF from place to place.

Binaries are important (at least to me, since their statistics formed part of "my" territory for a number of years). The problems start at the observational level, where theorists habitually use very incomplete studies of small samples of the incidence of duplicity and of the distributions of primary mass, mass ratio, period (or separation), and eccentricity. One cannot really extract the IMF from observations or define stellar populations by color or integrated line strength without allowing for them. On the theoretical side, one would like to know how they form, their relationship to triples and higher-order multiplicity (hierarchies vs. Trapezia and their stability), implications for numbers of planets, and their role in nucleosynthesis and other "advanced problems".

Disks and jets undoubtedly contribute (positively or negatively!) to planet formation, binary formation and other interactions, the disposal of spare magnetic flux and angular momentum, and a range of observable phenomena. My impression is that theorists have to work harder to make jets than disks. 
Global star formation rates are another area where the problems begin with getting hold of the data to be matched. One wants to know the SFR as a function of $z, Z$, and $z$, that is time (or redshift), metallicity, and location in galaxies or protogalaxies. General agreement seems to be limited to the rather qualitative - more at $z=1-3$ than now, but less farther back in time. I suspect Redman's theorem would then apply, that any competent theorist could explain the data using any theory. Large redshift is particularly important for reionizing the intergalactic medium so that we can see what is going on. Not that nature had any desire to help us, but there are at least a few clear sight lines back to $z=7-8$.

General vs. special purpose hardware and software. The hardware case was discussed at the symposium. Most star formation codes have been developed specifically for star formation, but I wonder whether there might be material we could steal from the (perhaps better funded) territories of cosmology/galaxy formation/VLSS and high energy astrophysics (which also involve turbulence, magnetic fields, and, of course, gravity!).

\section{Questions and Issues Arising from the Abstracts}

A large fraction of the titles and abstracts of both talks and posters gave the impression (which usually turned out to be correct) that the presenters intended to address one or more of the issues mentioned in Sect. 3. This is a good thing, and if it had not turned out to be the case, the strong implication would be that I was the wrong person to be given this task! But there were also a good many other items, sometimes more detailed, of which this is an attempted list, meaning that if you look hard in the proceedings you can probably find out something about them.

I have ordered these in the same pattern as the questions of Sect. 3, but as "NASA bullets" since many are fairly short. Interesting items mentioned in the original abstracts that did not find their way into the posters and talks as presented have been omitted.

\section{Initial Conditions}

- Measured magnetic fields are clearly important, but if determined from continuum polarization can be badly wrong if dust grains have been oriented by dynamical torques. An anonymous conference participant, acting as a referee, indicates that several theorists have concluded that at least the larger grains will be well aligned with the field in any case. Zeeman broadening of $\mathrm{OH}$ lines is an alternative field indicator. This is perhaps the place to mention, after the fact, that I had the great pleasure of being the (nonanonymous) referee of Prof. Larson's contribution, for which my only suggestion (quickly adopted) was full citations of the references.

- One also needs initial conditions for calculations of chemistry (next subtopic).

- Fluctuations in temperature and density of gas and dust can be described by thermodynamic considerations.

\section{Molecular Clouds}

- Accurate chemical evolution of the molecular gas is essential if observers' clouds and clumps (traced with $\mathrm{CO}, \mathrm{NH}_{3}, \mathrm{CH}_{3} \mathrm{OH}$, etc.) are to be compared with theorists' clouds and clumps (generally identified as density enhancements).

- The gas can be bistable; HI is correlated with total gas and star formation rates, though typically not as well as $\mathrm{H}_{2}$.

- Formation of GMCs from more extended HI is at least partly a fragmentation process in a multiphase ISM, whose dynamical evolution continues between the initial cloud and final proto-star cluster phases. 
- Filamentary clouds may yield a different IMF from spherical ones, and some real clouds are at least non-spherical in a way that may be related to triggering

- Collisions of superbubbles from previous star formation episodes can form molecular cloud complexes between them.

\section{Triggering}

- Run-away OB stars are another possibility, as is UV irradiation, from them or other sources (perhaps even AGNs?). Photoevaporative triggering can reveal itself in brightrimmed molecular clouds.

- Compressive tides could either trigger star formation or shelter gas from star formation.

\section{Star Clusters}

- Newly formed clusters are still embedded in dust and gas, and it is possible we may never see the real, unevolved IMF for O stars

- R Associations (with no OB stars but the later types obviously young) exist.

- Clusters can form around an initial very massive star (with triggering in a sort of ring).

- Dynamical segregation occurs gradually on the same time scale as the higher masses die.

- Galaxy mergers (etc.) can both trigger formation of star clusters and destroy existing ones.

- Comparisons of statistics of clusters and molecular clouds is one way of assessing the efficiency of star formation.

\section{Dynamic Range}

- Some indication of the difficulty of the problem is given by the extreme messiness of real protostellar envelopes (just think of the Vela and Rosette Nebulae).

\section{Star Populations}

- The acronym IRDC takes a moment to translate into InfraRed Dark Clouds. The real curiosity is that some of them actually have protostellar cores in them, and so are not really "starless cores".

\section{The Initial Mass Functions}

- No one really claimed that these are all the same, everywhere, though there is some disagreement about whether particular regions with young stars are "top heavy". These include the nucleus of our own galaxy (where one does not see the X-rays expected if there are lots of young, low-mass stars to go with the massive ones in the Arches etc.), 30 Doradus (where a normal IMF would probably tie up more than the total stellar mass of the LMC), and young massive clusters (which will end up as globular clusters in 10 Gyr or so only if they have an appropriate complement of low masses).

- Evolution of cores to stars in a cluster environment may be different enough to affect the IMF. A central black hole (for instance for star formation in disks around AGNs) is likely to inhibit formation of small stars, while having lots of dust should favor extra cooling and a smaller Jeans Mass for the turnover of the IMF.

\section{The Largest and Smallest Masses}

- Star growth by collisions in accreting cluster cores is expected.

- Accurate computations of the spectra expected for brown dwarfs as a function of mass and age are essential for recognizing them when they are not in binaries. 


\section{Binary Stars}

- Accretion can continue from circumbinary disks.

- Binary populations among very low mass stars and brown dwarfs are different from the A-K stars, though portions of this will reflect observational selection effects and dynamical evolution.

\section{Disks and Jets}

- Jets must somehow be launched, and get loaded up with molecular gas.

- Disks must disappear on the time scales set by observations (and models exist for making this happen either faster or slower than seen).

- There will be a dust sublimation front where we find dust-free disks.

\section{Global Star Formation Rates}

- High Velocity Clouds probably bring in fresh gas to enable star formation to continue. They themselves seem to contain no stars.

- Downsizing - the shift of the bulk of star formation to smaller galaxies as we approach the present - is observationally real and not readily reproduced in bottom-up models of galaxy formation and evolution.

- Something must terminate the formation of stars in early, massive galaxies (arguably the ionization of the gas so that the Jeans mass becomes some enormous number)

- In the case of population III, line pressure radiation on incoming gas (nearly pure $\mathrm{H}$ and $\mathrm{He}$ ) is not sufficient to prevent accretion, which, however, should stop with the UV breakout, ionization of the nearby gas, and radiation pressure from continuum photons.

\section{Hard- and Soft-ware}

- A number of presentations featured specific codes and machines on which they run, but my initial thought that we might be able to "borrow" things from the cosmology/galaxy formation community was stretched to include the high energy astrophysics community by a specific poster abstract, which mentioned disks, jets, magnetic fields and all, and was not spotted by the SOC as pertaining to neutron stars rather than YSOs until the program had been largely assembled.

\section{Things I Hadn't Thought of (but Should Have)}

- The most important of these is undoubtedly feedback - the effects of protostellar and stellar jets and all the rest on the surrounding gas, where they will certainly be a source of turbulence and, perhaps, of magnetic field amplification.

- Specific YSOs, star formation regions, star clusters, and merging galaxies mentioned in the presentations include the Perseus Arm (a reminder that spiral shock waves can be another trigger for GMC assemblage and collapse), Herbig-Haro objects, FUOr's (and EXor's, which by NOT having EX Ori as their prototype are just there to make things more difficult), the Antennae galaxies, NGC 3603, the Aquila Rift complex, Eta Cha, the Pipe Nebulae, AFGL 5142, Orion KL, NGC 1333, V4046 Sgr, Herbig Ae stars, Sh2-104, IRAS17149-3916, and V 582 Aur. This is not a complete list, which is one of the reasons the editors intend to provide a "object index" to this volume. 


\section{Some (Partial) Answers from the Symposium}

This is a subset. The 43 hours of talks (plus 10 I spent reading posters) would take an equal time to retell, just as you can really simulate a $10^{80}$ particle universe only with an $N=10^{80}$ calculation. The ones here are a personal, prejudiced choice.

\subsection{Binary Stars}

These are important for nucleosynthesis and for the use of Type Ia supernovae as distance indicators in cosmology. Whether Population III stars had no binaries or practically nothing but binaries has been determined - theoretically - both ways.

The desired data are numbers as a function of primary mass, mass ratio (my own little territory), period (or separation) and eccentricity, as a function of metallicity and formation epoch. The data change: population II when I was your age had "no" binaries. Globular clusters now have a large excess of X-ray binaries among other things. $N(e)$ is a convolution of formation and dynamical evolution, revealed by distributions of eccentricity vs. orbit periods in clusters of different ages.

$N\left(M_{1}\right)$ is intermediate for OB stars, high (more than $50 \%$ ) for A-K stars, and low again for M, L, and T types, reflecting formation, survival, and observational selection. One speaker suggested that nearly all stars form as triples, generally ejecting one member to leave binaries and singles about equally numerous. I think I last heard this at a 1979 meeting (Harrington 1982). $N\left(M_{1}, M_{2} / M_{1}, a\right)$ is clearly not a separable function. In fact, I suspect that all the distributions are actually sums of about 3 separate curves, arising from 3 different formation mechanisms, and simply blended in most data.

As for $N(Z, z)$, "there are very little data, and they are contradictory, so I will skip this point", quoting Lisa Deharveng on a different topic.

Common proper motion pairs can either be barely bound within clusters, or just drift off together.

One speaker remarked upon the difficulty of calculating the incidence of binaries in dwarf spheroidals (important because they must be allowed for in turning an observed velocity dispersion into a Virial mass to get $\mathrm{M} / \mathrm{L}$ for these nearly-invisible objects). Luckily observations come to our aid. Minor (2010, a thesis defended the week before the meeting) has found, with about a year of radial velocity data for several Milky Way companions, that there are binaries, some of which indeed have orbital velocities that can disturb $\sigma_{v}$, but that the effect on mass determination is generally modest, not exceeding $15 \%$ (but, of course, systematic, not random). A major factor is that large amplitudes come from two stars of nearly equal mass, which show themselves as double lines in the spectrum most of the time.

\subsection{Efficiency}

One speaker described the efficiency of star formation as the elephant in the conference room, because many calculations find a few percent to perhaps $15 \%$, and yet clusters remain bound when the residual gas leaves. That the average efficiency must be small follows because the Galaxy is about one hundred rotation periods old, so that a given gas atom has had about 100 arm-crossing chances to find its way into a star, and yet gas remains.

Many calculations yield 1-3\%, while cluster survival requires 15-20\%. One poster pointed out that it helps if most of the stars form in the cloud core. Data on numbers of clusters vs. age are not as helpful in defining survival time as you might expect. 
Encounter shocks at $z=8-9$ apparently yield quite a high ratio of mass going into stars vs. cold gas coming in.

\subsection{Brown Dwarfs}

These exist (20 years ago they did not, observationally), but are past the peak of the IMF in all contexts so far examined, and so are not a major dark matter component. Binary brown dwarfs also exist, though they are relatively rare and mostly have small separations (surely some combination of formation physics, dynamical evolution, and selection effects). They might form as binaries in accretion disks, be left behind when gas leaves a star formation region, or be gently ejected before they have had time to sweep up their fair share of gas.

One would like to know whether they form a continuous population with the orphan planets or exoplanets in general.

\subsection{Items New to $\mathrm{Me}$}

The concept of sinks is apparently quite new, so I feel no guilt. The same thing happened when I was summarizing a conference on large scale structure soon after the concept of $\sigma_{8}$ had been introduced [it is the rms density fluctuation on a scale of $8 M p c$ for $H=100 \mathrm{~km} / \mathrm{sec} / \mathrm{Mpc}$. Its numerical value is about one.

Other items like this include star formation on filaments (like VLSSS in the cosmological context), halo globular clusters being correlated with their central black hole masses, which in turn are correlated with the mass in bulge stars. If this means all this stuff has come from captures, I am confused.

Some more new stuff - that jets wiggle both observationally and theoretically; that low mass pre-main-sequence stars are a good deal fainter than expected (implying slow or episodic accretion), and that there is the risk of a magnetic braking catastrophe, because some angular momentum must be left to form planets!

\subsection{The Core Mass Function vs. the Stellar Initial Mass Function}

It was suggested that, in different contexts (and so conceivably all true), there is no close relationship (because of ongoing accretion, outflow, etc.), that they differ by a factor three, that they have the same slope when magnetic fields are included (but are otherwise different), that they arrive from the sum of several processes, that the observed IMF is universal, but the CMF is not; or conversely.

\subsection{Extrema}

The prize for the shortest talk went to Andy McLeod. The most popular encounter was NGC 4038/39 and the most popular cluster NGC 3603 (notice that virtually all well-known star clusters and galaxies have names beginning with $\mathrm{N}$ or $\mathrm{M}$ ).

The first star formed at $z=42$ in 2004 (this is funny if you read certain kinds of low-brow literature), which has perhaps grown to 70 this year.

The identity, mass, and explanation for the most massive stars remain uncertain. Qualitatively, the luminosities of main sequence stars scale as about $M^{3}$, so that you hit the Eddington limit at about $100 M_{\odot}$ (which must surely depend on both $\mathrm{Z}$ and $\mathrm{z}$ ). Calculations achieved either 110 or $140 M_{\odot}$ in the star from a $480 M_{\odot}$ core, both calculations coming from the same group, and they explained that the simulation is ongoing. Observationally, we certainly don't see many stars in excess of those numbers (when angular resolution is adequate), but it is not certain whether the lack is real or merely having 
$N(\geqslant M)=N_{0} M^{-2.3}$ go to zero. Formation of these very massive stars may well involve all of accretion, Rayleigh-Taylor instabilities, and mergers.

\subsection{Miscellany}

We are once again reduced to NASA bullets to collect many short (often verbless) items.

$\mathrm{H}_{2}$ could be just a tracer (vs. the cause) of star formation, in the sense that cold, dense HI would do just as well. Larson admitted off-line that he had once turned off all molecule formation in his 1968 code, and it made very little difference. But of course we need molecules to observe, and when they begin to condense out on ice, the densest cores become very difficult to observe, yielding systematic errors in mass vs. time and temperature.

Bottom up star formation appeared in one presentation, as was popular back in about 1970. It will yield a power law (like the energies of cosmic rays) from a sort of selforganized criticality.

The metallicity to change Pop III to Pop II was $10^{-5}$ in one poster, but $10^{-2}$ in another. I would go with the smaller number.

Prototypes are rarely typical, as in the case of Algols, RR Lyrae stars, and Cepheids, so perhaps also for the Pipe Nebula (justifying investigation of other regions).

Gravity can be left out, said one presenter. Not from my universe, despite potentially favorable effects on the masses of astronomers.

Magnetic fields in star formation regions make things smoother, yielding fewer and more massive stars and later star formation, while with turbulence you get finer structure, earlier stars, more stars, and more low mass stars, but measurements of magnetic fields by Chandrasekhar-Fermi method can yield the wrong answer (with the fields coming out smaller than the real values, if there is lots of small-scale structure).

Red and dead? Yes, E and SO galaxies have gas; it is just too hot to make stars.

Do tidal dwarfs get dark matter? They had better if they are to be like the dwarfs in the Local Group, while tidal globular clusters had better not. One generally does not have masses for these young, very bright entities and so cannot tell what they will like in 10 Gyr.

Every trigger or driver of turbulence you have ever heard of is surely important somewhere - mergers, SN(R) shocks, HII expansion, outflow, runaway OB stars, Herbig Haro objects, shocks at arms, photoevaporation, UV radiation, external photoionization, and surely some we have not yet thought of.

\section{Looking Ahead}

If the past is Sir James Jeans stepping down as president of the Royal Astronomical Society in 1927, and the present is this 2010 conference, then the future means 2093 (which will be my 150th birthday). By then, studies of star formation will probably have bumped against some fairly fundamental limits.

On the observational side, CCDs already have something like $90 \%$ quantum efficiency, so "mehr licht" has to mean bigger mirrors. Angular resolution can improve as $\theta=\lambda / D$, but for some phenomena a sort of Olbers' Paradox will set in, with the sky completely covered by the things you want to study (for instance HI fluctuations or even dwarf galaxies at large redshift). Timing is limited by photon bunching on the nano-second scale (but much sooner by atmospheric scintillation, unless you take your 600-meter mirror into space). Spectral resolution is limited by the uncertainty principle and the 
length of time you are willing to count wave crests! Admittedly, this is a wavelength resolution somewhere around $10^{19}$ for optical astronomers.

On the computational side, Bruce Elmegreen looked at realistic futures. But, if you wish to look at unrealistic ones you could start with a thermodynamic limit that erasing one Gigabyte adds $S=8.2 \times 10^{-14} \mathrm{~J} / \mathrm{K}$ of entropy to the universe, and so requires dumping heat of $2.5 \times 10^{-11} J$ at room temperature. Make up your own rules for how many Gbyte per particle your N-body simulation will need, how many time steps are required for your version of the Courant condition, and how long you are willing to let your simulation run. I ended up with $4 \times 10^{26}$ particles per GWatt, while a mere star has $10^{57}$ particles. This is apparently not quite the way to go.

The computer of the future will presumably be some sort of quantum picodot, but it will still be I/O limited, as were the very early computers of 1954, when my father worked for NCR, then trying to rival IBM. (It lost.) My theoretical astrophysicist of 2093 is under copyright and cannot be shown here, but he is green and wears glasses over quite large eyes.

\section{Last Words}

Some of the discussion following papers made me feel that the phrase "star formation community" might be an oxymoron. This is not actually bad - it means that people in the field are sure enough of its importance and permanence to be able to afford to risk not always showing a united front.

"There are", said Anne B. Underhill, "more models that aren't stars than there are stars that aren't models". This still seems to be true, though she said it in 1967 at the Prague IAU (which was attended by at least one person at S270). Miriam Lichtheim was of the same generation and equally tart, but she taught me the basics of reading and writing Middle Egyptian at UCLA in 1962-63.

It is the traditional prerogative of the last speaker to express collective thanks to the local organizers (Josep Miguel Girart and his whole team) and the scientific program organizers (João Alves, Bruce Elmegreen and their team), and this I do both individually and collectively, most heartily.

My last overhead had the words for "farewell" or "until next time" in 20 languages, and it was a special pleasure to be able to add Korean, Turkish, Persian, Finnish, and three other I don't actually recognize to this list during the meeting.

\section{References}

Alves, J. F \& McCaughrean, J. J. Eds. 2001, The Origins of Stars and Planets, Springer ESO Astrophysics Symposia

Harrington, J. P. 1982, in M. H. Hart \& B. Zuckerman, Eds., Where Are They?, Pergamon Press p. 152 (from a 1979 meeting)

Hayashi, C. 1961, Publ. Astron. Soc. Japan, 13, 450

Jeans, J. 1935, Through Space and Time: The Christmas, 1933, Royal Institution Lectures, Macmillan

Larson, R. B. 1969, Mon. Not. Royal Astron. Soc. 145, 271

Minor, Q. 2010, Ph.D. Dissertation, U. California, Irvine, defended May 2010

Paczyński, B. 1970, Acta Astron. 20, 47

Sugimoto, D. 2010, Astron. \& Geophys. 51, 3.36, and personal communication

Tinsley, B. M. 1968, Astrophys. J. 151, 547 